\title{
A simple model for trading climate risk *
}

\author{
Sébastien Chaumont, Peter Imkeller and Matthias Müller \\ Institut für Mathematik \\ Humboldt-Universität zu Berlin \\ Unter den Linden 6 \\ 10099 Berlin \\ Germany \\ Ulrich Horst \\ Department of Mathematics \\ University of British Columbia \\ 1984 Mathematics Road \\ Vancouver, BC V6T 1 Z2 \\ Canada
}

May 11, 2005

\begin{abstract}
Short term climate events such as the sea surface temperature anomaly known as El Niño are financial risk sources leading to incomplete markets. To make such risk tradable, we use a market model in which a climate index provides an extra investment option. Given one possible market price of risk each agent can maximize the exponential utility from three sources of income: capital market, additional security, and individual risk exposure. Under an equilibrium condition the market price of risk is uniquely determined by a backward stochastic differential equation. We translate these stochastic equations into semi-linear partial differential equations for the simulation of which numerical schemes are available. We choose two simple models for sea surface temperature, and with ENSO risk exposed fisher and farmer and a non-exposed bank three toy agents. By simulating their optimal investment into the climate index we obtain first insight into the dynamics of the market.
\end{abstract}

\section{Introduction}

In recent years, a new type of financial products on incomplete markets has appeared at the interface of finance and insurance. Its purpose is securitization, i.e. to shift

${ }^{*}$ This work was partially supported by the DFG research center Matheon (FZT 86) in Berlin. 
certain (re-) insurance risks to the capital markets. The products we focus on deal with natural exterior risks generated in particular by weather and climate phenomena. The first product of this type was traded by the New York and the Chicago Mercantile Exchange and was called the New York HDD swap (see [9]). The demand for these products may come from energy companies supplying gas to retail distributors. If for example the heating season in winter is unusually warm, due to a smaller volume of gas sold profits may shrink. Another example is given by the risk due to big accumulative losses for example in farming or fishing caused by the most well known short term climate event of the El Niño Southern Oscillation (ENSO).

In this paper, we give a comprehensive report about a simple market model designed for climate risk sharing of agents exposed to this risk source, or more generally risks exogenous from the point of view of usual capital markets. In this simple model external risk becomes tradable by the key concepts of market completion in a partial equilibrium. Trading and hedging the risk makes no use of the classical insurance concept of predicting catastrophic events, and then arranging the risk capital at one instant of time in case the catastrophic event is predicted to happen with a certain threshold probability. The dynamic hedging approach we propose and describe below in more detail will rather be based upon the innovative concept of market composition by agents exposed in a negatively correlated or even complementary way to the climate risk. Due to the negative correlation of their exposure to risk, the total welfare of the agents participating in this redistribution market will increase. The calculations and simulations obtained so far will not be able to answer the question whether the welfare increase of dynamic hedging of complementary interests or the classical insurance approach's benefits prevail. This will require more quantitative studies, and precise concepts of the prediction quality along with the correlation structure. To illustrate our risk trading model, think of the toy agents used in this paper: a rice farmer profiting from El Niño conditions, a fisher suffering from them, and a risk neutral bank which is just interested in diversifying its portfolio.

The model we propose is discussed in detail in [12] and [7]. We consider an economy with a finite number of climate affected agents $a \in I$. In order to describe their risk exposure in an analytically accessible way, we represent the climate (sea surface temperature) process $K$ by simple low dimensional stochastic differential equations suggested by the climate physics literature. Among them are Ornstein-Uhlenbeck type models, or stochastic oscillators fluctuating randomly periodically between two metastable states.

The agents composing our market get a climate-exposed revenue from their usual business described by some payoff functional $H^{a}(K, X)$ depending on the climate process $K$ as well as on stock price process $X$. This stock price represents a stock market where they can buy and sell according to their preferences, without being able to change prices. The agents aim at hedging their revenue against random fluctuations. If they had risky payoffs $g(X)$ depending on the stock price $X$ alone, they could replicate them. In other words, they could find strategies for trading with the stock reaching $g(X)$ as terminal wealth. This optimal trading could then be described by the Black-Scholes formalism. In our setting, this instrument is insufficient: it is impossible to replicate a climate dependent risk exposure $H^{a}(X, K)$ using only the stock. The climate process 
has an uncertainty inherent in the risk exposure and modelled by a Wiener process $W^{2}$ that is independent of the Wiener process $W^{1}$ modelling the stock market uncertainty inherent in $X$. The stock market is therefore incomplete and we are well beyond the Black-Scholes realm.

In order to make hedging of the risk exposure $H^{a}(K, X)$ possible, we complete the market by constructing a special security $Y$ modelled by means of $W^{2}$, the climate risk uncertainty process. Together with this special security, the market possesses assets covering all relevant uncertainty processes. It therefore is complete, and climate risk becomes tradable. Agents active on the market may buy or sell individual amounts of this climate index according to their random risk exposures. So the agents have three sources of income stemming a) from their the risk exposure $H^{a}(K, X)$, b) from trading with the stock $X$ and c) from trading with the special security $Y$. Given a particular candidate of a price process $Y$, every agent chooses an investment strategy which optimizes the individual utility from his total wealth from trading and his random income subject to climate risk. There will be a unique price process $Y^{*}$ for which a market clearing for climate risk is achieved, i.e. for which there is zero excess demand for the climate index. This pricing rule is determined by a backward stochastic differential equation (BSDE).

Besides existence and uniqueness of the partial equilibrium it is difficult to get quantitative analytical results about the model. For this reason we use numerical simulations on the level of quasi-linear Partial Differential Equation (PDE) to obtain insight into the market's quality and dynamics. This becomes possible, since BSDE are related to this class of PDE via the Feynman-Kac link.

The paper is organized as follows. In section 1 we give a brief account of the ENSO phenomenon and its economic consequences. Reduced ENSO models we employ for our analysis are explained in section 4, while the market model based on completion and partial equilibrium is discussed in 3 , and in more mathematical details in 4 . In 5, we obtain some quantitative insight into the market dynamics by treating some toy examples in numerical simulations. Section 5 suggests an equilibrium approach to pricing temperature bonds and outlines avenues for future research.

\section{Economic consequences of the ENSO phenomenon}

The ENSO phenomenon has been known to Peruvian fishermen before the arrival of the Spaniards through its spectacular economical effects. The normal large scale of atmospheric pressure distribution over the Southern Pacific shows a zone of high pressure over the eastern part near the South American coast, while a zone of lower pressure prevails over the western part of the ocean. This pressure difference on sea level is expressed in the so-called Southern Oscillation Index (SOI) which is usually positive. Positive SOI forces trade winds to blow east to west. At randomly periodic times - every 3-8 years (the El Nino years) - however, the SOI becomes negative forcing the trade winds to relax or even blow in the reverse direction. Ocean currents are largely influenced by trade winds at sea level. In particular, the Humboldt Current along the South American coast may be affected. It normally transports cold water northward. 
During an El Nino event, the relaxation of trade winds allows warm water to appear on the surface of the Southern Pacific near the South American coast (see Figure 1).
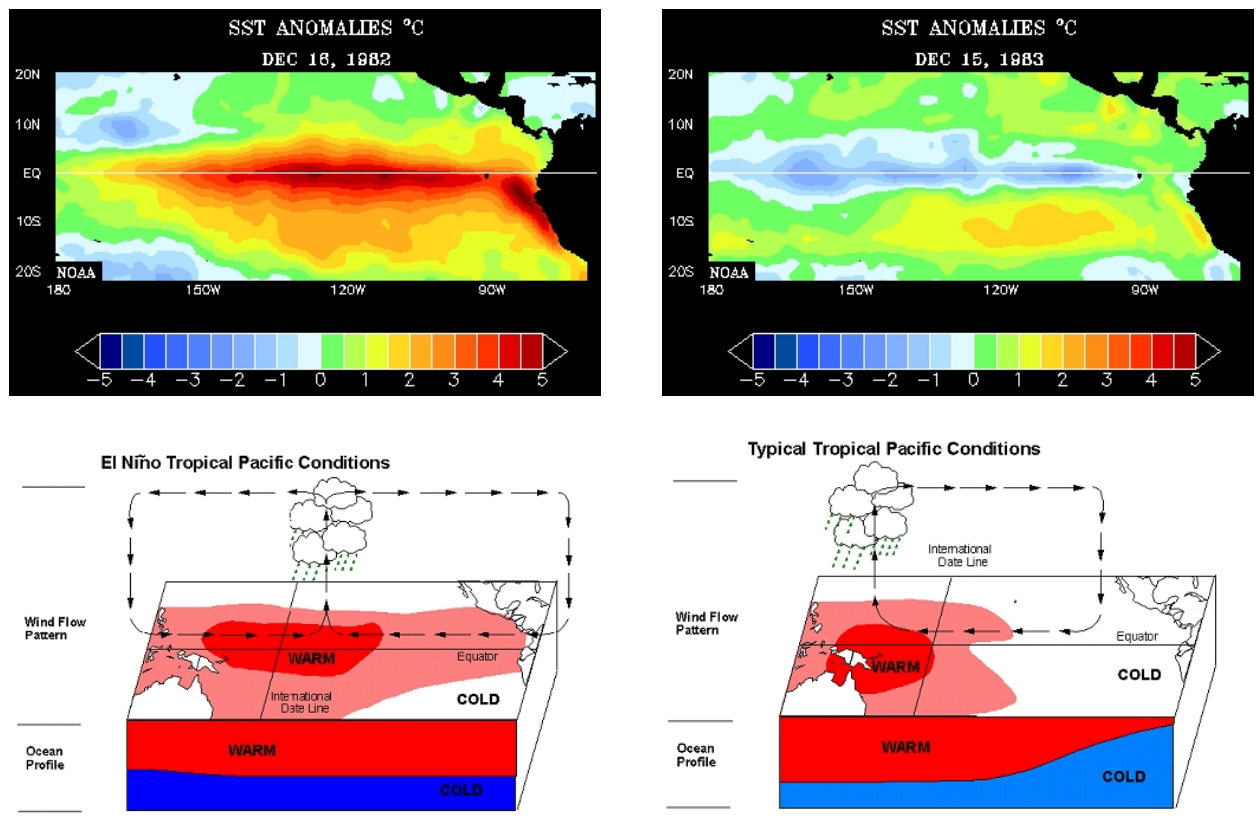

Figure 1: El Niño versus normal conditions, source: NOAA, www.jam-web.de/el-nino

The effects of this change of the sea surface temperature on marine life are tremendous. The trade wind shift disrupts the upwelling of oxygen and nutrient rich cold water, one of the basic conditions for dense concentrations of marine life. Let us give some numbers first for the local effect on the Peruvian fishing industry. As in most developing countries in the tropics with economies depending largely on few branches for example in food production, the sensitivity to climatic fluctuations is very high. According to a study of the World Resources Institute (1994), El Niño contributed to the collapse of the Peruvian fishing industry. From the early 1950s through 1971 the harvest increased, peaking at more than 12 million tons per year. With the arrival of the 1972/1973 El Nino, a disastrous drop of the harvest to 2.5 million tons was recorded (see Figure 2).

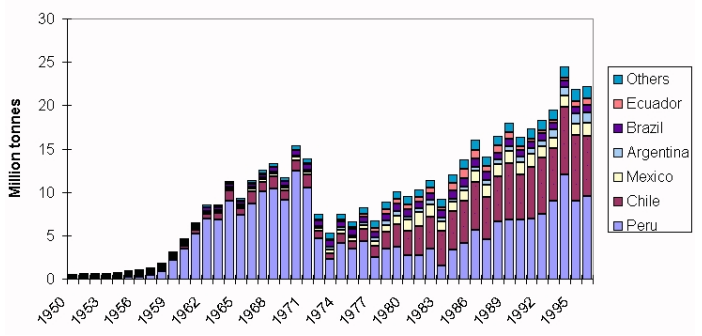

Figure 2: Peruvian anchoveta catch rates, source: www.fao.org

The consequences of this distortion of ocean currents due to changes in the SOI are much more global than one may conjecture at first glance. The change of the tropical 
Pacific sea surface temperatures induced by the fluctuation of trade winds affects the atmosphere in turn directly by causing convection. Dense tropical rain clouds are created which, besides increasing the amount of precipitation the western hemisphere receives, distort the atmospheric air flow in altitudes of $5-10 \mathrm{~km}$ above sea level. One global pattern becomes clearly visible: in El Nino periods, rain areas usually centered above Indonesia and the far western Pacific move eastward into the central Pacific, which affects waves in the tropospheric air flow causing unusual weather over many regions of the globe (see Figure 3).

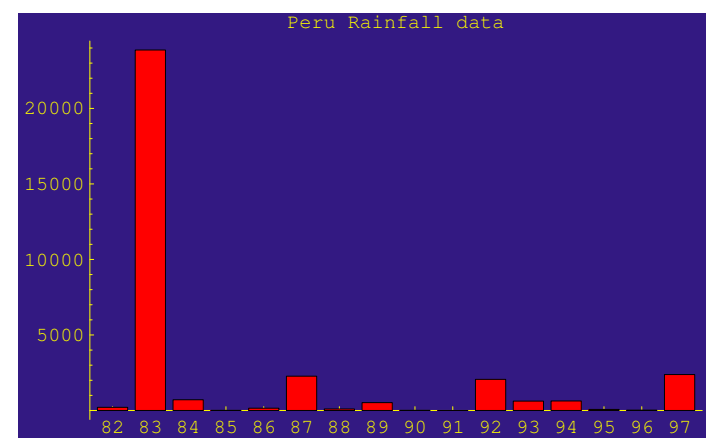

Figure 3: Precipitation in Peru and El Niño, source: tao.atmos.washington.edu

The climatic effects of ENSO create globally and even locally groups of possible agents on markets which are affected in a very different, sometimes even complementary, way. Let us illustrate this by giving some examples. As explained above, American fishing industries from Peru to Canada are strongly affected during El Nino years by seriously dropping catch numbers. Quite opposite changes are observed on the other "pole" of the Southern Oscillation. According to Gaol and Manurung [11] and Mizuno [21], catch numbers for big eye tuna in the South Java sea waters, one of the most important tuna fishing regions of the world, during El Niño periods increase by about 30 percent, due to an opposite effect on the sea surface temperatures in the Western South Pacific (see Figure 4). Another pair of groups of economic agents with complementary interests is given by farmers and fishers even in the same national economy. Warm El Niño years are unfavorable for fishers for the reasons given, but may be favorable to farmers in parts of the country normally dry due to increased amounts of precipitation. Cold years usually following in the heels of El Niño years are welcomed by fishermen, but not by farmers, because of droughts and crop failures. For example, rice and cotton, two of the primary crops grown in Northern Peru, are highly sensitive to the quantities and timing of rainfall.

\section{Some simple ENSO models}

For the conceptual models we have in mind that are designed to distribute the ENSO risk or more generally any market external risk among affected agents, we need simple models able to describe the main features of the climate process involved. In our case this is the random process describing the sea surface temperature in the Southern 

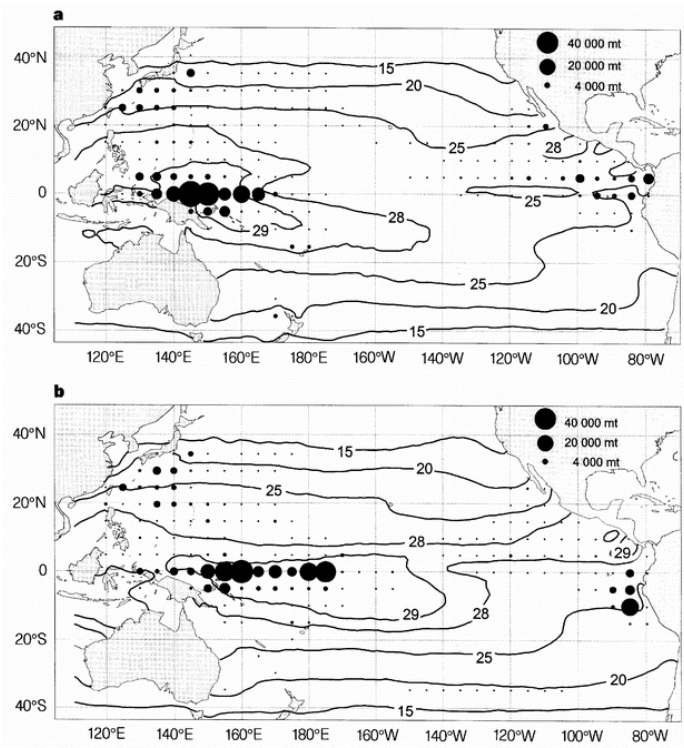

Figure 4: "Skipjack" tuna catch rates, source: Nature, vol. 389 (1997)

Pacific. It is usually modeled as a one-dimensional stochastic process. The reduced physical models it originates from usually lead to finite dimensional stochastic equations and describe some nonlinear interaction between finitely many physical quantities including the local temperature. There are around 15 reduced models for ENSO, of which we briefly sketch 3. For example, the stochastic differential equation model by Wang, Barcilon and Fang [1] describes a nonlinear interaction between two physical quantities: the thermocline depth in some area of the South Pacific, i.e. the depth of the surface layer $h$ of the ocean that can be considered as physically active, and the sea surface temperature $K$, with a two dimensional stochastic perturbation $\left(W^{1}, W^{2}\right)$ interpreted by the wind forcing of the sea surface. The equation is given by

$$
\begin{aligned}
d K_{t} & =a_{1} K_{t}-b_{1} K_{t}^{3}+c_{1} h_{t}+d W_{t}^{1}, \\
d h_{t} & =a_{2} h_{t}-b_{2} h_{t}^{3}+c_{2} K_{t}+d W_{t}^{2},
\end{aligned}
$$

where $a_{1}, \cdots, c_{2}$ are real parameters. The system turns out to be an autonomous nonlinear stochastic oscillator which in some parameter regimes acts as a stochastically perturbed bistable differential equation with an intrinsically defined periodicity. Typical trajectories of the temperature component $K$ relevant for our purposes show stochastic bi-stable behavior (see Figure 5).

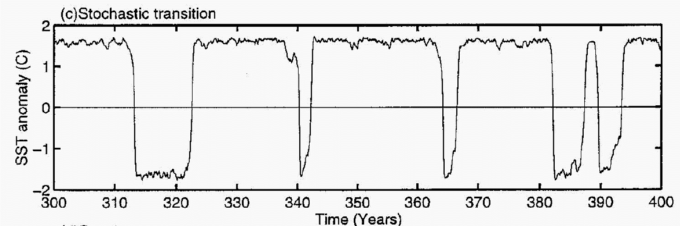

Figure 5: Bistable SST process, source: [1] 
Most of the time they fluctuate in the vicinity of one or the other of two meta-stable equilibria, interrupted by spontaneous rapid transitions between the domains of attraction of these states. The transitions follow some randomly periodic pattern, which actually is the reason why they are considered as depicting the qualitative behavior of the temperature evolution of the Pacific sea surface characteristic for ENSO. For simulation purposes later, to retain these qualitative features, we take a one-dimensional Stochastic Differential Equation (SDE) driven by a Brownian motion. It describes the motion of a state variable traveling through a bi-stable potential landscape, with an explicit periodic dependence of the potential shape creating a non-autonomous stochastic system.

Our second example (see Penland [23] comes from a 15-dimensional linear SDE of the Ornstein-Uhlenbeck type

$$
d X_{t}=B X_{t} d t+\sigma d W_{t}
$$

with a $15 \times 15$-matrix $B$ with non-trivial rotational part and entries determined by satellite measurements, and a stochastic driving term of intensity $\sigma$. This equation is obtained from an empirical orthogonal function development fitted to time series of observations over several decades of recent climate history. This fitting shows that 10-20 orthogonal functions are needed, which leads to the dimensionality of the linear Ornstein-Uhlenbeck type stochastic equation. It appears to be the reduced model most frequently used for ENSO predictions. Mathematically it creates a diffusion with nontrivial rotation numbers implying random periodicity for the sea surface temperature variable $K$ which can be generated through the vector $X$. To retain the qualitative features of the development of $K$ for simulation purposes, we may describe it as a simple mean-reverting linear SDE with an additional deterministic periodic forcing.

A third, very simple but equally interesting qualitative example was discussed by Suarez, Schopf [25] and by Battisti [4]. Here, the feedback on the temperature exerted by the thermocline depth in the first model is short-cut and leads to a one-dimensional stochastic differential equation with time delay of the form

$$
d K_{t}=\left[a K_{t}-b K_{t}^{3}+c K_{t-\Delta}\right] d t+d W_{t} .
$$

Here $\Delta$ is a fixed delay length, $a, b, c$ real parameters, and $W$ again a Brownian motion describing wind forcing of the sea surface (see Figure 6).



Figure 6: Delay model for SST

The delay effect in this equation may also be interpreted alternatively. Variations in the thermocline depth near the South American coast triggered by the ENSO rise of 
sea surface temperature create Kelvin waves that travel across the Pacific, get reflected at the Asian coast, travel back, and impact the system again with its state delayed by the total travelling time.

After setting up a simple financial market model to deal with distributing ENSO risk in a way considered as optimal by the individual agents interested in trading it, we do numerical simulations of their optimal investment strategies. The climate component we use in these simulations, besides mean-reverting Ornstein-Uhlenbeck processes, will be the above mentioned one-dimensional non-autonomous stochastic hopping between two meta-stable states of a diffusion travelling in a simple potential landscape with two wells, the relative depth of which alternates periodically. This model constitutes the paradigm of stochastic resonance. See [14] for a review. More formally, its main component is a potential function $U$ with a double well, for example $U(k)=\frac{k^{4}}{4}-\frac{k^{2}}{2}, k \in \mathbb{R}$. The diffusion process $K$ given by the SDE

$$
d K_{t}=U^{\prime}\left(K_{t}\right) d t+Q K_{t} \sin \left(\frac{2 \pi}{T} t\right)+\sigma d W_{t}
$$

models temperature in a bi-stable environment. For the noise intensity $\sigma$ and the periodicity parameter $Q$ chosen appropriately, the trajectories of $K$ are almost periodic, with a random periodicity fluctuating around $T$ (see Figure 7 ).

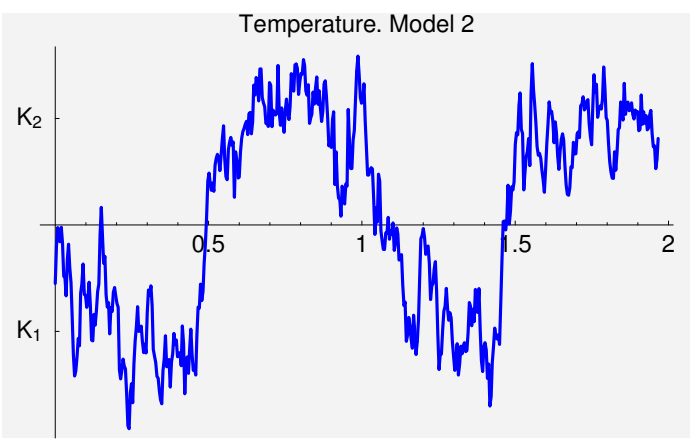

Figure 7: Brownian diffusion in a double well potential

\section{$3 \quad$ An equilibrium model for risk trading}

We now explain the market model we create in order to enable agents whose total income depends on ENSO to relocate their individual risk to other parts of the market. We consider an economy with a finite number of small agents $a \in I$, i.e. agents unable to influence the price dynamics of the market, represented for instance by individual farmers or fishers, banks, or insurance companies. The model only allows for a redistribution of agents' individual risks, optimal with respect to their individual preferences. The main advantage of this model is that the market is ideally composed of agents with complementary interests. The principal idea is that a redistribution of risk among them may be better than a classical insurance approach in which the prediction of the risky event causing high accumulative losses is used for adjusting 
risk capital for instance. In fact, climate events usually are embedded into nonlinear models, for which the frequently used linear prediction mechanisms have considerable shortcomings. At this place, we should mention that our model is able to deal with any risk source located in the exterior of a classical stock market.

Our agents are typically exposed to a climate process $K$ described by one of the simple models discussed in the previous section. For our simulations we shall use the phenomenological randomly periodic bi-stable temperature process.

The agents composing our market are allowed to have three sources of income, and trade continuously within a time interval $[0, T]$. To be able to realize all of these components in the simplest possible setting, we fix a stochastic basis $\left(\Omega,\left(\mathcal{F}_{t}\right)_{0 \leq t \leq T}, P,\left(W^{1}, W^{2}\right)\right)$ composed of a two-dimensional Wiener space $\left(W^{1}, W^{2}\right)$. Think of $W^{1}$ as describing the uncertainty inside a usual stock market, while $W^{2}$ is the driving uncertainty in the climate process $K$.

Firstly, the agents can trade on a financial market. In the simplest possible case which we are going to sketch, the financial market consists of a bond and a risky asset. We take the bond as a trivial constant process with interest rate 1 , and assume that the stock price process $X$ is of the type of a geometric Brownian motion. Formally, it is determined by the stochastic differential equation

$$
d X_{t}=X_{t}\left[b_{t}^{X} d t+\sigma_{t}^{X} d W_{t}^{1}\right]=X_{t} \sigma_{t}^{X}\left[d W_{t}^{1}+\theta_{t} d t\right]
$$

where the drift $b^{X}$ and the volatility $\sigma^{X}$ are smooth random functions, that are constant in the classical case. Following usual terminological habits, the process $\theta$ can be called the price of market risk.

The second source of income of agent $a$ is a risky individual function $H^{a}(K, X)$ determined by his exposure to climate $K$ given by

$$
d K_{t}=b_{t}^{K} d t+\sigma_{t}^{K} d W_{t}^{2}
$$

and also depending on the stock price process $X$. In the simplest case, we only think of shuffling climate risk within the market of affected agents. For our simulations in this setting, we consider a toy market with two or three toy agents, represented by ENSO affected farmer (farming company) and fisher (fishing company), plus eventually a bank not directly exposed to climate. Consequently, three typical types of qualitative risk exposure will be considered. Recall our model climate process $K$ showing randomly periodic bistable behavior by hopping between a low $\left(K_{1}\right)$ and a high $\left(K_{2}\right)$ meta-stable state. The former one corresponds to usual conditions for sea surface temperature of the Southern Pacific, while the second one represents ENSO conditions. So the fisher may have his temperature of optimal income near the lower equilibrium, while the farmer might profit more from higher precipitation rates at the higher temperature equilibrium. This in particular means that the fisher profits from temperature values under which the farmer suffers most, and vice versa. The exposure of the bank is taken to be independent of $K$. More formally, we arrive at the following simple exposure models. 
Fisher. Take a fisher $f \in I$ that makes most profits if the temperature is near $K_{1}$. We thus can describe his income $H^{f}$ during the period $[0, T]$ qualitatively by

$$
H^{f}=\int_{0}^{T} \varphi^{f}\left(K_{s}\right) d s,
$$

where $\varphi^{f}$ is a positive function taking its global maximum in $K_{1}$, for example (see Figure 8)

$$
\varphi^{f}(k)=e^{-\left(k-K_{1}\right)^{2}}
$$
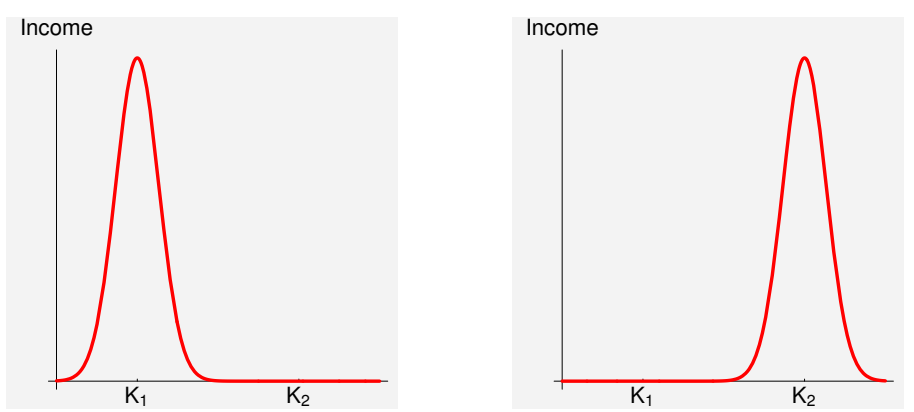

Figure 8: Risk exposure for fisher (left) and farmer (right)

Farmer. The (rice) farmer is taken to have an exposure of the same type as the fisher. The optimal income is just obtained at $K_{2}$, the second meta-stable point of a bi-stable process $K$. The income of the farmer may therefore be described by

$$
H^{r}=\int_{0}^{T} \varphi^{r}\left(K_{s}\right) d s
$$

where $\varphi^{r}$ is a positive function taking its global maximum in $K_{2}$, for example

$$
\varphi^{f}(k)=e^{-\left(k-K_{2}\right)^{2}} .
$$

If we work with a bi-stable $K$, we see immediately that farmer and fisher have complementary interests, and therefore are likely to profit from trading the climate risk among each other.

Bank. As an additional agent, we consider a bank $b$ whose profits only come from its portfolio management from investment on the financial market, and which participates in the climate risk share only to diversify its portfolio. So its exposure functional will be the trivial $H^{b}=0$.

Due to the presence of the uncertainty factor $W^{2}$ which is independent of the uncertainty factor $W^{1}$ governing the stock market, the risks represented by $H^{a}$ cannot be hedged on the stock market alone: we face a typical incomplete market situation. We deal with this problem by a technique of market completion. We introduce and add to the market a security $Y$, through which climate risk becomes tradable and which therefore acts as the third source of income. Agents active on the market may buy or sell individual amounts of this climate index according to their random risk exposures. 
The price process for this climate index of course has to be driven by the climate uncertainty. Formally, $Y$ is again described by a geometric Brownian motion type process given by the equation

$$
d Y_{t}=Y_{t}\left[b_{t}^{Y} d t+\sigma_{t}^{Y} d W_{t}^{2}\right]=Y_{t} \sigma_{t}^{Y}\left[d W_{t}^{2}+\eta_{t} d t\right]
$$

with smooth drift and volatility processes $b^{Y}, \sigma^{Y}$. Following and extrapolating general habits, we shall call $\eta$ the price of climate risk. Of course, this price is not fixed from the beginning, but has to be determined by the dynamics of the market, i.e. by a market equilibrium in which all the agents' individual preferences are respected, and optimized.

We can proceed in two steps to achieve such an equilibrium. Suppose first that a possible candidate $\eta$ for this price of climate risk is given. Now every agent $a \in I$ trades on the completed market, so that his individual preferences are optimally fulfilled. This will lead him to an individual optimal investment strategy. We describe the preferences by an exponential utility function with individual risk aversion, and assume that the agent optimizes this utility from terminal wealth obtained through his three sources of income. To achieve a market equilibrium in a second step, we now have to choose a particular $\eta *$ for which, if each agent has acted optimally according to the rules of the first step, the market is cleared of the amount of climate index $Y$ available. This equilibrium price of climate risk $\eta *$ is uniquely determined, as will be further discussed. Hence in our model the external risk dynamically determines in a unique way the market price of risk of the climate index $Y$ via the risky incomes, the preferences and the partial market clearing condition.

\section{The market dynamics and its equilibrium}

Let us now explain in a somewhat more detailed way how the equilibrium of our climate risk trading market is obtained. First of all, we have to make precise the individual agents' trading according to their preferences. Each agent $a \in I$ is supposed to be endowed with an initial capital $v_{0}^{a} \geq 0$. If a possible candidate $\eta$ for the equilibrium price of climate risk is fixed, he invests in the market including the climate index, and to do so, he uses admissible trading strategies $\pi=\left(\pi^{a, X}, \pi^{a, Y}\right)$. This basically means that the wealth processes, resulting from investment along these strategies, are well defined. $\pi^{X}$ is the proportion invested in the financial market asset $X$, while $\pi^{Y}$ is related to investment into the climate index $Y$. Therefore $a$ 's wealth process is given by the stochastic differential equation

$$
V_{t}^{a, \eta}=v_{0}^{a}+\int_{0}^{t} \pi_{s}^{a, X} \frac{d X_{s}}{X_{s}}+\pi_{s}^{a, Y} \frac{d Y_{s}}{Y_{s}}, t \in[0, T] .
$$

Each agent $a$, by acting on the trading strategies $\pi$ available to him, wants to maximize the expected utility of the sum of the terminal wealth $V_{T}^{a}$ from investment into income sources $X$ and $Y$, as well as the risky income $H^{a}$ subject to climate exposure. His preferences are described by an individual exponential utility function

$$
U^{a}(x)=-\exp \left(-\alpha_{a} x\right), x \in \mathbb{R}
$$


with individual risk aversion coefficient $\alpha_{a}>0$. Now $a$ tends to maximize his terminal wealth measured by exponential utility. In mathematical terms, he wants to attain

$$
J^{a}=\sup _{\pi \text { admissible }} \mathbb{E}\left[U^{a}\left(V_{T}^{a, \eta}+H^{a}\right)\right] .
$$

Under simple assumptions (see [12]), this quantity can be computed via well known techniques based on duality and Legendre transforms for $U^{a}$. See Karatzas, Lehoczky, Shreve [16] or Kramkov, Schachermayer [20]. They are most easily applicable, if applied from the perspective of the risk neutral measure $Q^{\eta}$ under which the world is seen in the direction of the the drifts $(\theta, \eta)$ figuring in the price processes $(X, Y)$. In other words, under $Q^{\eta}$, the processes $W^{\eta}=\left(W^{1}+\int_{0}^{\cdot} \theta_{s} d s, W^{2}+\int_{0}^{\cdot} \eta_{s} d s\right)$ are Brownian motions. Under regularity assumptions verified in our context, it is well known that $Q^{\eta}$ is absolutely continuous for $P$, and the density is given by

$$
\frac{d Q^{\eta}}{d P}=Z^{\eta}=\exp \left[-\int_{0}^{\cdot}\left(\theta_{s} d W_{s}^{1}+\eta_{s} d W_{s}^{2}\right)-\frac{1}{2} \int_{0}^{\cdot}\left(\theta_{s}^{2}+\eta_{s}^{2}\right) d s\right]
$$

Under $Q^{\eta}$, the optimal individual utility can be calculated via duality, and the optimal strategy is described using conditioning. We have

$$
J^{a}=\mathbb{E}\left[-\frac{\lambda_{a}}{\alpha_{a}} Z_{T}^{\eta}\right]=-\frac{\lambda_{a}}{\alpha_{a}} .
$$

where $\lambda_{a}$ is determined by initial wealth, more precisely by

$$
\log \left(\lambda_{a}\right)=\log \left(\alpha_{a}\right)-\alpha_{a} v_{0}^{a}+\mathbb{E}^{\eta}\left[-\log \left(Z_{T}^{\eta}\right)-\alpha_{a} H^{a}\right] .
$$

The optimal income agent $a$ obtains from trading in the two securities and his exposure to external risk therefore depends on the market price of external risk $\eta$. Denote the optimal trading strategy of agent $a$ by $\pi^{a, \eta}=\left(\pi^{a, \eta, X}, \pi^{a, \eta, Y}\right)$. Since $Z^{\eta}$ is exponential, and the formula for the computation of optimal utility involves taking logarithms, a formula comparing the terminal income from the three sources due to optimal investment and the density structure reads

$$
\begin{aligned}
c_{a}+ & \frac{1}{\alpha_{a}}\left(\int_{0}^{T}\left(\theta_{s} d W_{s}^{1}+\eta_{s} d W_{s}^{2}\right)-\frac{1}{2 \alpha_{a}} \int_{0}^{T}\left(\theta_{s}^{2}+\eta_{s}^{2}\right) d s\right. \\
& =H^{a}+\int_{0}^{T}\left(\pi_{s}^{a, \eta, X}\left(d W_{s}^{1}+\theta_{s} d s\right)+\pi_{s}^{a, \eta, Y}\left(d W_{s}^{2}+\eta_{s} d s\right)\right) .
\end{aligned}
$$

We conclude that each individual agent's maximal utility from terminal wealth depends on the process parameter $\eta$, the price of climate risk. To determine a unique price of risk $\eta^{*}$ under which the market reaches an equilibrium for which every agent obtains his maximal income we impose a partial market clearing condition. It states that the total investment $\left(\pi^{a, \eta, Y}\right)_{a \in I}$ in the insurance asset satisfies the condition

$$
\sum_{a \in I} \pi^{a, \eta, Y}=0 \text { a.s. }
$$


We use this condition in (5) by summing it over $a \in I$. Then, to find $\eta^{*}$ and the corresponding unique risk neutral measure $Q^{\eta^{*}}$, we have to extend the resulting equation dynamically to all times $t \in[0, T]$. As shown in [12], Theorems 3.3 and $3.5, \eta^{*}$ emerges as the solution of a nonlinear BSDE (backward stochastic differential equation). More precisely, with the abbreviations

$$
\begin{aligned}
\bar{\alpha} & =\left(\sum_{\alpha \in I} \frac{1}{\alpha_{a}}\right)^{-1}, \quad \bar{H}=\sum_{\alpha \in I} H^{a}+\frac{1}{2 \bar{\alpha}} \int_{0}^{\tau}\left(\theta_{t}\right)^{2} d t \\
z_{1} & =\theta-\bar{\alpha} \sigma^{X} \sum_{a \in I} \pi^{a, \eta, X}, \quad z_{2}=\eta,
\end{aligned}
$$

we are led to a nonlinear BSDE of the form

$$
h_{s}=\bar{\alpha} \bar{H}-\int_{s}^{T} z_{t} d W_{t}-\int_{s}^{T} \theta_{t}^{S} z_{1, t} d t-\frac{1}{2} \int_{s}^{T} z_{2, t}^{2} d t
$$

to be solved for the process $\left(h, z_{1}, z_{2}\right)$. It is shown in [12] that this equation possesses a unique solution which yields a unique partial equilibrium $\eta^{*}$ of the market. Conversely, if the market has a partial equilibrium, is is given by a (unique) solution of the BSDE (7). This approach uses results from Kobylanski [19]. This settles existence and uniqueness questions for solutions of the climate risk securitization problem in a partial market equilibrium. Apart from this, it is difficult to derive analytical results, for instance about structure properties of the equilibrium price, or the dynamics and quality of the market.

\section{Numerical simulation of the market}

In this section we add a brief discussion on how to compute and simulate the optimal investment strategies $\pi^{a, \eta, Y}$ into the climate index $Y$ in equilibrium. We shall look at simple scenarios of exposure of few agents to climate risk. We know from the previous section that the equilibrium price can be obtained as the solution of a BSDE with quadratic nonlinearity. Yet, the numerical analysis of BSDE is still in its infancy (see for example [5]). Therefore, we chose to transfer our stochastic simulation problem into a problem of simulating non-linear PDE. In fact, via the generalized FeynmanKac formalism, BSDE are associated with systems of linear or semi-linear parabolic partial differential equations. Their solutions exist in general in the viscosity sense, much as in Chaumont [6]. In the simple situations we consider, they turn out to be classical. We use newly developed numerical schemes for non-linear PDE from [6] to approximate and simulate them for Ornstein-Uhlenbeck type or bistable diffusion climate processes, and the risk functionals for fishers, farmers and bank as described in section 3. This way we obtain first information on the dynamics of such a market which will, if not quantitatively, be of interest at least for qualitative issues.

Here is a brief outline of the link between systems of forward-backward stochastic differential equations and quasi-linear PDE, which in the linear case boils down to the 
usual Feynman-Kac formalism. Let $X^{t, x}, t \in[0, T], x \in \mathbf{R}$ is the family of diffusion processes starting in $x$ at time $t$, given by the stochastic equations

$$
d X t, x_{s}=b\left(X_{s}^{t, x}\right) d s+\sigma\left(X_{s}^{t, x}\right) d W_{s}
$$

with infinitesimal generator

$$
L f=\frac{1}{2} \sigma^{*} \sigma f^{\prime \prime}+b f^{\prime}
$$

Let $F$ be a smooth function of the real variables $x, y, z$, which is taking the role of the nonlinear part. Suppose that a smooth function $v$ solves the nonlinear PDE

$$
-\frac{\partial v}{\partial s}-L v-F\left(v, \sigma^{*} v^{\prime}\right)=0
$$

Then, if we put

$$
\begin{aligned}
Y^{t, x} & =v\left(\cdot, X^{t, x}\right), \\
Z^{t, x} & =\sigma v^{\prime}\left(\cdot, X^{t, x}\right),
\end{aligned}
$$

the pair $\left(Y^{t, x}, Z^{t, x}\right)$ solves the nonlinear BSDE

$$
d Y_{s}^{t, x}=-F\left(X_{s}^{t, x}, Z_{s}^{t, x}\right) d t+Z_{s}^{t, x} d W_{s}
$$

In general, $v$ is only a viscosity solution. In the case of our climate risk trading, we have $F(v, z)=\frac{1}{2} z^{2}$. Using this link, we transform the BSDE of the previous section into the associated PDE. Then we employ numerical schemes approximating the solutions of these parabolic quasi-linear PDE. Following Chaumont [6], a method initiated by Barles and Souganidis [3] based on the well known stability results for viscosity solutions (see [8], [2] for a general presentation) will be applicable to our schemes and allow to simulate optimal investment strategies of some toy agents in a simple and small market.

\section{$5.1 \quad$ Toy models}

We use the following models.

\section{Model A}

The time horizon is chosen to be $T=2$. We use an Ornstein-Uhlenbeck process to describe the climate process $K$, with the following coefficients :

$$
d K_{s}=-K_{s}+\frac{1}{2} d W_{2, s}, \quad s \in[0, T] .
$$

Here we consider only two model agents, a fisher and a bank as described in section 3 . The fisher's random income function is

$$
H^{f}=\int_{0}^{T} \varphi^{f}\left(K_{s}\right) d s,
$$

with $\varphi^{f}(k)=5 \exp \left(-10 k^{2}\right)$, for all $k \in \mathbb{R}$. This means that the optimal temperature for the fisher is normalized to be 0 . The bank has no risky income, i.e. $H^{b}=0$. We assume that each agent uses the risk aversion coefficient $\alpha^{f}=\alpha^{b}=1$. 


\section{Model B}

The temperature is now modelled by a periodically forced bi-stable temperature process with coefficients

$$
d K_{s}=-8\left(K_{s}^{3}-K_{s}\right)-\sin (2 \pi s)+4.5 d W_{2, s}, \quad s \in[0, T] .
$$

See Figure 7 for a sample path of this process.

Again, we choose $T=2$ for the time horizon, i.e. 2 periods of the temperature process. This process is close to the high meta-stable equilibrium $K_{2}=2.5$ for $t \in$ $[0 ; 0.5] \cup[1 ; 1.5]$ and symmetrically close to the low value $K_{1}=-2.5$ for $t \in[0.5 ; 1] \cup$ $[1.5 ; 2]$. Again we consider only two agents, a fisher and a farmer with respective income

$$
H^{f}=\int_{0}^{T} 5 \exp \left(-10\left(K_{s}-K_{1}\right)^{2}\right) d s \text { and } H^{r}=\int_{0}^{T} 5 \exp \left(-10\left(K_{s}-K_{2}\right)^{2}\right) d s,
$$

where the optimal temperature is $K_{1}=-2.5$ for the fisher and $K_{2}=2.5$ for the farmer, which coincide with the bistable states of the temperature process. We again assume that each agent uses the risk aversion coefficient $\alpha^{f}=\alpha^{r}=1$.

\section{Model C}

This model uses the same characteristics as model B except for the time horizon, which is now chosen to be $T=3 / 2$, i.e. 3 half periods of the temperature process $K$. This gives an advantage to the farmer, since the temperature spends 1 unit of time i.e. $2 / 3$ of the trading interval near the meta-stable state favorable for the farmer, and only 0.5 units of time near its low meta-equilibrium favorable for the fisher.

In all the models, the share price is a geometrical Brownian motion with very strong coefficients $b^{S}=1$ and $\sigma^{S}=1$.

\section{$5.2 \quad$ Simulated optimal strategies}

Since only two agents are active on the market, the local equilibrium condition (6) implies that at each time $t$ the entire quantity of $Y$ sold by one agent is bought by the other, i.e.

$$
\pi^{f, \eta^{*}, Y}=-\pi^{b, \eta^{*}, Y} \text { in model A, or } \pi^{r, \eta^{*}, Y}=-\pi^{f, \eta^{*}, Y} \text { in models B and C. }
$$

Therefore it will be enough to show diagrams of the strategy of one agent (fisher in model $\mathrm{A}$ and farmer in models $\mathrm{B}$ and $\mathrm{C}$ ).

We show the optimal strategies as functions of $t$ (on the period $[0,1]$ ) and the current temperature $K_{t}$. The diagrams also display the optimal amount of money to be exchanged between the agents, from the selected agent's point of view.

\subsubsection{Model A}

Here we only show the fisher's optimal strategy $\pi^{f, \eta^{*}, Y}$. At the optimal temperature for fishing $K_{t}=0$, the fisher makes his maximal profit, and we observe that there is no exchange of risk trading money. As soon as the temperature grows a little, the fisher has to buy a certain quantity of $Y$ from the bank. This exchange will bring security to the fisher and profits to the bank. 

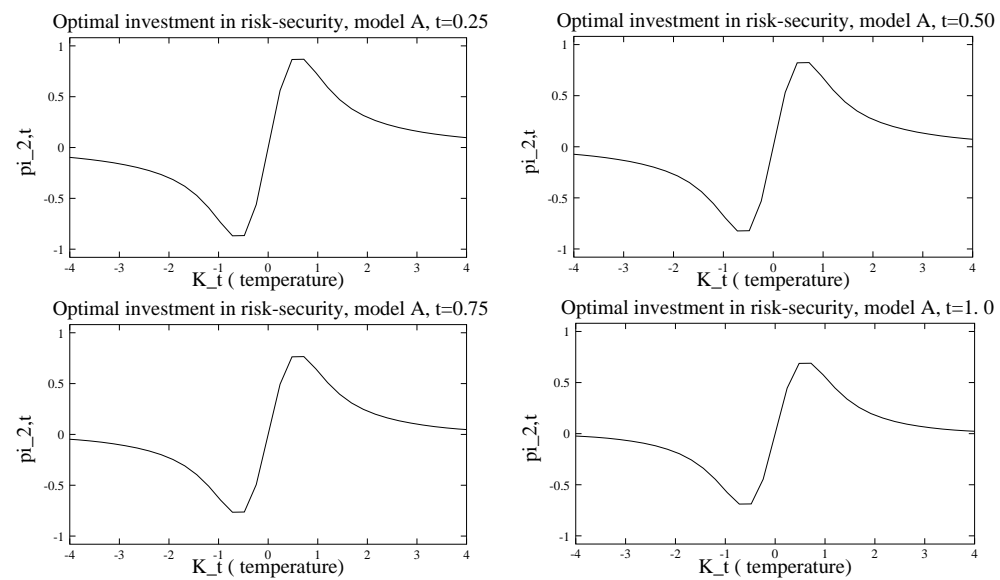

Figure 9: The optimal strategy for the fisher (model A).

\subsubsection{Models $\mathrm{B}$ and $\mathrm{C}$}

We only show the farmer's optimal strategy $\pi^{r, \eta^{*}, Y}$. In our diagrams, we see that the farmer invests in $Y$ as long as the temperature is high in the first half period $[0,0.5]$, i.e. an interval that favors him, and sells $Y$ to the fisher as long as the temperature is low, for $t \in[0.5,1]$, i.e. when he needs money. This corresponds well to the intuition that the agents have an interest to share their risks by exchanging money this way. The qualitative difference between models $\mathrm{B}$ and $\mathrm{C}$ is not big. We just observe that the farmer invests a little more than the fisher.
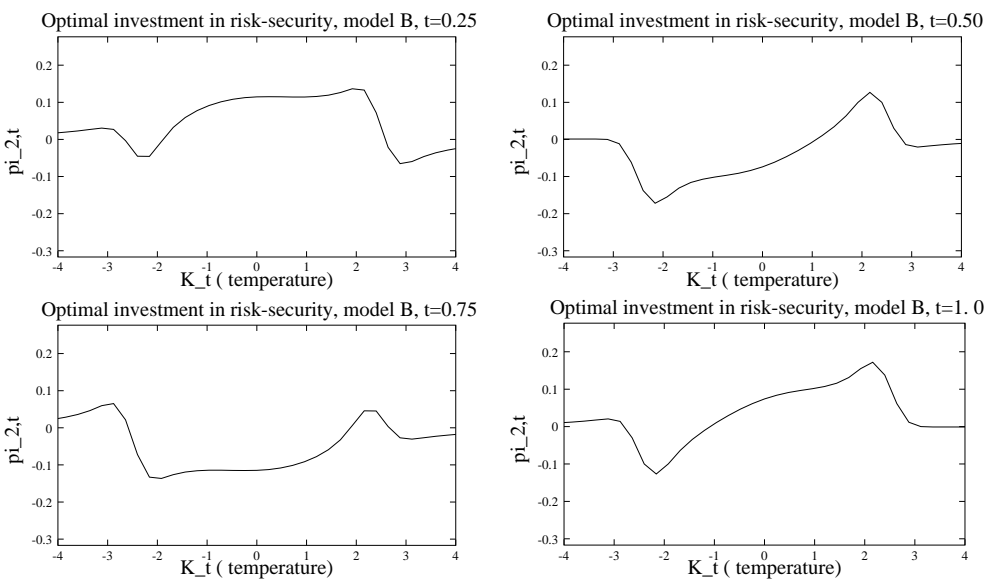

Figure 10: The optimal strategy for the farmer (model B).

\section{Outlook: Equilibrium pricing of climate bonds}

This section briefly illustrates how the approach of market completion can be extended to pricing and hedging weather sensitive securities. Insurance companies have long provided protection against extreme meteorological events. While these companies have a 

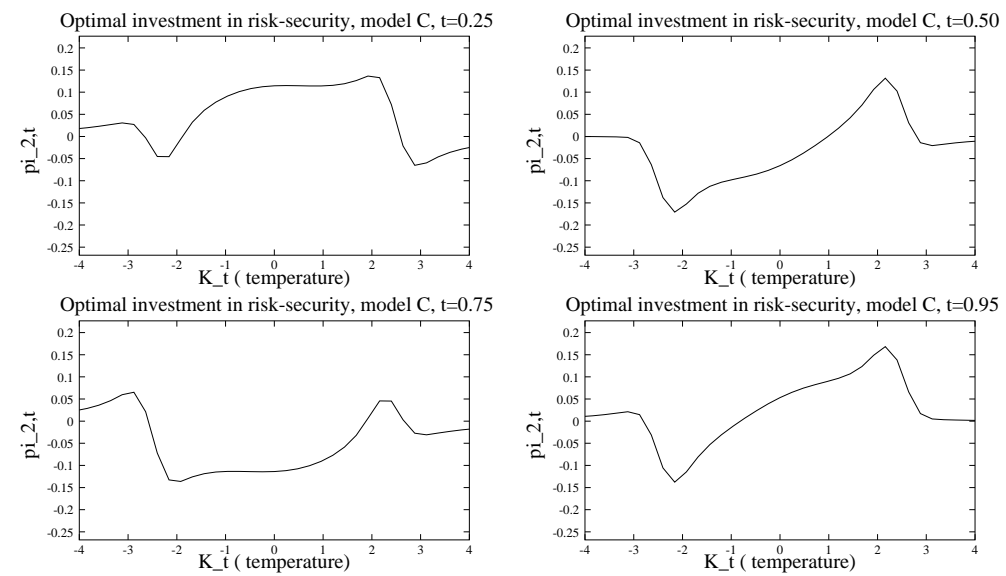

Figure 11: The optimal strategy for the farmer (model C).

variety of instruments available to hedge their asset portfolios, until recently the only way to hedge their underwriting risk was to lay off a part of it with reinsurers. Despite its desirable characteristics, traditional reinsurance has its limitations. Insurance companies too often retain large exposures to catastrophic events which can easily overtax individual companies. For instance, in the 1990s a devastating earthquake in Northbridge (CA) left nine insurance companies bankrupt, and two large California insurers unwilling to further write earthquake coverage.

Insurance companies trying to transfer their climate related risk to capital markets need to transform non-tradable risk into tradable financial securities. One option is to issue bonds whose terminal payments $H_{I}(K)$ depend on some climate process $K$. Such a bond will typically be traded among agents exposed to climate risk and possibly by institutional investors seeking to diversify their portfolio by means of financial securities that have no correlation with capital market indices. Pricing climate-related securities does not follow actuarial approaches characteristic of insurance contracts, but is rather based on financial valuation principles. However, these securities are typically written on non-tradable assets such as temperature indices. They contain uncertainty components independent of the ones inherent in the market's risky assets, and therefore escape tradability through the latter, making the market incomplete. This renders the traditional Black-Scholes framework based on market completeness inappropriate a benchmark model for pricing and hedging weather derivatives. In addition, the market for climate derivatives is usually illiquid. One should thus bring in techniques from equilibrium theory when pricing weather bonds.

Given a market price $\eta$ of climate risk, the bond price $B_{t}^{\eta}$ at time $t$ is given by the bond's conditional expected terminal payoff,

$$
B_{t}=\mathbb{E}_{t}^{\eta}\left[H_{I}\right]
$$

where the expectation is taken with respect to the risk neutral pricing measure $Q^{\eta}$ under which $W^{2}$ is a Brownian motion with drift $\eta$. Assuming that the bond completes the market and that the agents' preferences can be described by exponential utility functions, optimal trading strategies $\bar{\pi}^{a, \eta}=\left(\bar{\pi}_{t}^{a, \eta}\right)$ in the bond exist. In equilibrium, 
the market for the bonds clears, i.e., in equilibrium the market price of risk $\eta^{*}$ satisfies

$$
\sum_{a \in I} \bar{\pi}^{a, \eta^{*}}=0 \text { a.s. }
$$

and the bond price process is given by (11) with $\eta=\eta^{*}$. By analogy to the models described in Section 4, the process $\eta^{*}$ can be characterized by the solution of a BSDE.

While it is an open problem to state general conditions on the bond's payoff structure and the agents preferences and risk exposures that guarantee that the additional security completes the market, in certain situations this assumption can indeed be verified. In the simplest case we may think of an insurance company selling protection against unusually warm temperatures during the holiday season to operators of ski resorts at some premium $P$. If at some predetermined date $T$ the temperature exceeds a certain threshold $\underline{k}$, the policy holder is entitled to a cash payment $C$ proportional to $K_{T}-\underline{k}$ up to a maximal amount proportional to $\bar{k}-\underline{k}$, i.e.,

$$
C:=c\left\{\left(K_{T}-\underline{k}\right)^{+}-\left(K_{T}-\bar{k}\right)^{+}\right\} .
$$

One possibility of laying off some of the insurance risk to financial markets consists in issuing a bond with terminal payoff

$$
H_{I}=\max \{\alpha P-C, 0\} \quad(p \in[0,1]) .
$$

Simplifying even further, we assume that the temperature process $K$ is modeled by a mean-reverting Ornstein-Uhlenbeck process of the form (10) and that the bond is traded among farmers $a \in I$ that suffer from a financial loss $H_{a}(K)$ if the temperature $K_{T}$ falls below some threshold $k_{0}$. More specifically, these agents are exposed to a financial loss of the form

$$
H_{a}(K)=c_{a}\left\{\left(k_{0}-K_{T}\right)^{+}-\left(k_{1}-K_{T}\right)^{+}\right\}
$$

where $k_{0}<k_{1}$. Since $H_{I}$ is negatively correlated to the agents' income, their risk exposure can be reduced by trading the newly issued climate bond. Using modifications of arguments given in [22], one can indeed show that the bond with terminal payoff $H_{I}$ completes the market and that a unique equilibrium pricing measure $Q^{\eta^{*}}$ exists under which the bond price process takes the form (11). An extension of our equilibrium approach to more general climate securities such as HDD swaps and the question of how to optimally design the bond's payoff structure remains a topic for future research. 


\section{References}

[1] A. Barcilon, Z. Fang, B. Wang, Stochastic dynamics of El Nino-Southern Oscillation. J. Atmos. Sci. vol. 56, IPRC-31 (1999), 5-23.

[2] G. Barles, Solutions de Viscosité des Equations de Hamilton-Jacobi. Mathématiques et Applications 17, Springer Verlag (1994).

[3] G. Barles, P. E. Souganidis, Convergence of Approximation Schemes for Fully Nonlinear Second Order Equations. Asymptotic Analysis vol. 4, Elsevier Science Publishers B.V. North-Holland (1991), 273-283.

[4] D. S. Battisti, On the role of off-equatorial oceanic Rossby waves during ENSO. J. Phys. Oceanography 19 (1989), 551-559.

[5] B. Bouchard, N. Touzi, Discrete time approximation and Monte-Carlo simulation of backward stochastic differential equations. Stoch. Proc. Appl. ?? (2004), ??

[6] S. Chaumont, Gestion optimale de bilan de compagnie d'assurance. PhD Thesis, Université Nancy I (2002).

[7] S. Chaumont, P. Imkeller, M.Müller, Equilibrium trading of climate and weather risk and numerical simulation in a Markovian framework. Submitted, HU Berlin (2004).

[8] M. G. Crandall, H. Ishii, P.-L. Lions, User's guide to Viscosity Solutions of 2nd Order PDE. Bulletin of the American Mathematical Society, vol. 27(1) (1992), $1-67$.

[9] M. Davis. "Pricing weather derivatives by marginal value". Quantitative Finance 1 (2001), 1-4.

[10] J. Cox, C. F. Huang, Optimal consumption and portfolio policies when asset prices follow a diffusion process. J. Econ. Th. 49 (1989), 33-83.

[11] J. L. Gaol, D. Manurung, El Nino Southern Oscillation impact on sea surface temperature derived from satellite imagery and its relationships on tuna fishing ground in the South Java seawaters. AARS (2000).

[12] Y. Hu, P. Imkeller, M. Müller, Partial equilibrium and market completion. Preprint, HU Berlin (2003).

[13] S. Herrmann, P. Imkeller, The exit problem for diffusions with time periodic drift and stochastic resonance. Preprint, HU Berlin (2003).

[14] S. Herrmann, P. Imkeller, I. Pavlyukevich, Stochastic resonance: non-robust and robust tuning notions. Preprint, HU Berlin (2003).

[15] P. Imkeller, I. Pavlyukevich, Stochastic resonance: a comparative study of twostate models. Preprint, HU Berlin (2002). 
[16] I. Karatzas, J. P. Lehoczky, S. E. Shreve, Optimal portfolio and consumption decision for a small investor on a finite horizon. SIAM J. Contr. Optim. 25 (1987), $1157-1586$.

[17] I. Karatzas, J. P. Lehoczky, S. E. Shreve, Existence and uniqueness of multi-agent equilibrium in a stochastic, dynamic consumption/investment model. Math. Oper. Res. 15 (1990), 80-128.

[18] I. Karatzas, S.E. Shreve, Brownian Motion and Stochastic Calculus. Springer Verlag (1988).

[19] M. Kobylanski, Backward stochastic differential equations and partial differential equations with quadratic growth. Annals of Probability vol. 28(2) (2000), 558-602.

[20] Kramkov, D., Schachermayer, W. The asymptotic elasticity of utility functions and optimal investment in incomplete markets. Ann. Appl. Probability 9 (1999), 904-950.

[21] K. Mizuno, Variabilities of Thermal and Velocity Field of North of Australia Basin with Regard to the Indonesia Trough Flow. Proceedings of the International Workshop on Trough Flow Studies in Around Indonesian Waters, BPPT, Indonesia (1995).

[22] M. Müller, Market Completion and Robust Utility Optimization. PhD Thesis, Humboldt University Berlin (2005).

[23] C. Penland, A stochastic model of Indo Pacific sea surface temperature anomalies. Physica D 98 (1996), 534-558.

[24] S. Pliska, A stochastic calculus model of continuous trading : optimal portfolio. Math. Oper. Res. 11 (1986), 371-382.

[25] M. J. Suarez, P. S. Schopf, A delayed action oscillator for ENSO. J. Atmos. Sci. 45 (1988), 3283-3287. 